\title{
Dimensionamiento de Tanques Biorreactores mediante Simulación Computacional
}

\author{
José de Souza(1), Clayton A. Oliveira-Motta(1), Lirio Schaeffer ${ }^{(1)}$, Elton Gimenez-Rossini( ${ }^{(2)}$ \\ (1) Universidad Federal de Rio Grande del Sur, Programa de Posgrado en Ingeniería de Minas, Metalurgia y \\ Materiales, Av. Bento Gonçalves, 9500, Barrio Agronomia, CP 91501970 Porto Alegre, RS-Brasil. \\ (e-mail: josesouza@liberato.com.br, clayton.motta@yahoo.com.br, schaefer@ufrgs.br) \\ (2) Universidad Estadual de Rio Grande del Sur - UERGS, Polo NH, Curso de Ingeniería en Energía, \\ Calle Inconfidentes, 395, Barrio Primavera, Novo Hamburgo, RS-Brasil (e-mail: elton-rossini@uergs.edu.br)
}

Recibido Jul. 10, 2014; Aceptado Sep. 23, 2014; Versión final recibida Nov. 11, 2014

\begin{abstract}
Resumen
Este trabajo tiene el objetivo de dimensionar un biorreactor de forma optimizada. El dimensionamiento correcto y la elección de materiales son ítems imprescindibles para garantizar un buen funcionamiento y rendimiento de la planta para la generación de biogás. Las herramientas computacionales pueden ayudar en el proyecto y dimensionamiento de plantas de biogás. En este trabajo se han ejecutado simulaciones computacionales para conocer el espesor mínimo de las paredes del biorreactor, considerando tres aceros: AISI 318, AISI 316 L y ASTM A36. La simulación de los biorreactores para dimensionamiento y la elección de los materiales se han desarrollado con el uso de software de CAE FEMAP NASTRAN 10.0.2. Los resultados muestran que el material óptimo es el acero AISI 318 mientras que los aceros AISI $316 \mathrm{~L}$ y ASTM A36 tuvieron un comportamiento semejante en la simulación.
\end{abstract}

Palabras clave: biogás, biorreactores, simulación, diseño, selección de aceros

\section{Dimensioning of Bioreactors Tanks by Computer Simulation}

\begin{abstract}
The objective of this work is to optimize and scale a bioreactor. The design and choice of materials are essential aspects to ensure proper operation and performance of the plant for biogas generation. Computational tools can assist in the design and sizing of biogas plants. Computer simulations were performed to determine the minimum plate thickness of the bioreactor considering three types of steels: AISI 318, AISI 316 L y ASTM A36. The simulation of bioreactors for the design and for the choice of materials is done using the software CAE FEMAP NASTRAN 10.0.2. The results show that the optimum material is steel AISI 318 while the steels AISI 316 L y ASTM A36 presented a similar behavior during simulation.
\end{abstract}

Keywords: biogas, biorreactors, simulation, design, steel selection 


\section{INTRODUCCIÓN}

El metano que proviene del biogás representa una fuente de energía cada vez más importante en áreas rurales, debido en parte al incremento de la construcción de plantas de biogás en regiones de producción de alimentos de Alemania. Todo material orgánico representa una importante fuente de combustible en potencia porque se puede convertirlo en energía (Souza; Schaeffer, 2010). El modelado y simulación con softwares CAD 3D es un medio de dimensionar y modelar piezas, partes y conjuntos para facilitar el proceso de fabricación y montaje de plantas de biodigestión y, además, permiten analizar una serie de aspectos involucrados en este proceso. Algunas herramientas computacionales proveen soluciones para la planificación y concepción de instalaciones complejas, líneas de montaje y locales de trabajo. En el contexto de prototipaje digital se pueden usar dichas herramientas para reducir el ciclo de desarrollo de productos (Chablat et al., 2010). Sectores económicos y energéticos mundiales están concentrando inversiones en tecnologías para la producción de biocombustibles (Cervi et al., 2011). La sustitución de los hidrocarburos por fuentes alternativas sostenibles de generación de energía se ha transformado en una prioridad en los últimos años (Morero y Campanella, 2013). La filtración del biogás (reducción de gases no combustibles) transforma el mismo en biometano. Este posee contenidos de metano $\left(\mathrm{CH}_{4}\right)$ en torno a un $90 \%$. Se pueden utilizar los microorganismos para la purificación de biogás. Los mismos pueden remover las substancias consideradas contaminantes, especialmente para la eliminación de sulfuro de hidrógeno (Varnero et al., 2012).

Gran parte de las plantas de biogás en Europa y EUA utilizan biorreactores cilíndricos en posición vertical. En Alemania, en 2009, ese modelo totalizaba cerca de un $90 \%$ del total de plantas de biogás (Oechsner y Lemmer, 2009). Dichos biorreactores poseen reservatorio con fondo rígido de hormigón y paredes de acero, hormigón armado o aún materiales especiales, como polímeros rígidos PEAD (Polietileno de alta densidad) (Souza; Schaeffer, 2013). Puede ser parcial o totalmente enterrado, o ser posicionado completamente sobre el suelo. En todos los modelos, la parte principal se compone del tanque, el biorreactor. El mismo es responsable por la degradación del substrato (Souza, 2010). Dichos tanques son dimensionados en función de la carga orgánica, o sea, de la cantidad de material orgánico que se pretende tratar. Del punto de vista económico, las plantas de biodigestión necesitan de biorreactores de fácil fabricación, montaje y manutención. Los biorreactores en aleaciones de acero inox tienen ventajas porque ofrecen buena resistencia contra agentes corrosivos. La estructura posee condiciones apropiadas para la instalación de agitadores o mezcladores, divisorias, mecanismos de calentamiento y control de temperatura (Coelho et al., 1999). El formato rígido y fijo es propicio para revestimientos térmicos y protectores. Dicho sistema posee aspectos favorables para la instalación de válvulas para el control de entrada y salida del substrato de biogás (Iglesias et al., 2000).

Con una gran cantidad de aplicaciones, las herramientas y tecnologías para estudios en el área de la simulación, como softwares computacionales, pueden simular eventos relacionados con movimiento, estudios específicos que involucran alto costo de fabricación, estructuras químicas, mecánicas, entre otras. En la mecánica, la simulación de los dispositivos móviles y de su manipulación facilita la visualización de la apariencia, interferencias, posicionamiento, etc. Esta evaluación reduce costos y facilita ajustes, además de tener fácil utilización (Guerra, 2012). Para efectuar la simulación computacional para optimización de tanques, ha sido necesario observar el código ASME (American Society of Mechanical Engineers) que tiene el objetivo de estandarizar, asociar y consolidar las diversas normas existentes para construcción de tanques y recipientes a presión. La norma ASME considera que las tensiones admisibles para determinado material son las máximas tensiones adoptadas para el dimensionamiento de un recipiente a presión. Dichas tensiones deben ser menores que los límites de resistencia y elasticidad del material en la temperatura de proyecto considerada. La fabricación de tanques como los biorreactores deben observar el código ASME Sección VIII - División 1.

\section{METODOLOGÍA}

Para este trabajo, se ha dimensionado un biorreactor con volumen de $2.128,7 \mathrm{~m}^{3}$; en el análisis se han considerado tres materiales diferentes para comparase a través de simulación computacional. Las características dimensionales del biorreactor son: diámetro: $22.000 \mathrm{~mm}$; altura total del costado: $6.000 \mathrm{~mm}$; altura de columna de agua: $5.600 \mathrm{~mm}$. En la figura 1 se puede ver el modelo de biorreactor de biogás con paredes de acero atornilladas en anillos y cúpula para componer gasómetro en lona de goma. El tanque con $6.000 \mathrm{~mm}$ de altura y diámetro de $22.000 \mathrm{~mm}$ forma parte de un proyecto de una planta de biogás. Empleado en plantas de biogás para retención y degradación del sustrato y para generación de biogás. El sustrato permanece en promedio 30 días en el biorreactor en fermentación. Los biorreactores representan un porcentual considerable del costo de la planta: en torno a un $60 \%$. 


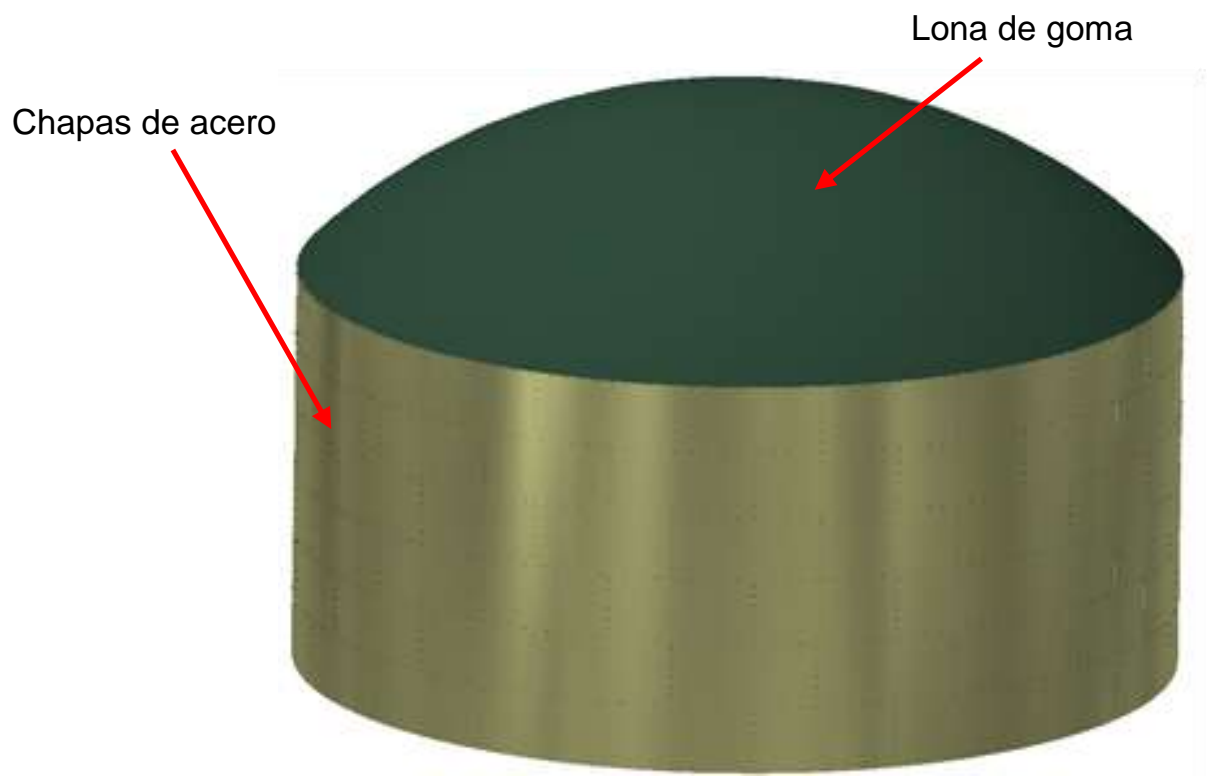

Fig. 1: Tanque biorreactor utilizado en plantas de generación de biogás.

Se ha utilizado el software Inventor Professional 2013 para modelaje y dimensionamiento del biorreactor, y FEMAP Lastran 10.0.2 para simulación computacional del mismo. Se han elegido tres materiales metálicos de aleación distintos para análisis y comparación: acero dúplex AISI 318, que es un material todavía nuevo en el mercado; acero austenítico AISI 316L, inoxidable, muy utilizado en la fabricación de tanques para materiales corrosivos; y acero carbón laminado ASTM A36, indicado por el código ASME para fabricación de recipientes a presión. Se han elegido los primeros aceros porque ya han sido analizados en ambiente biocorrosivo y se utilizan en la fabricación de biorreactores. También el acero carbón es objeto de investigación para su utilización en biorreactores con vitrificación de las chapas.

Para el cálculo de la presión, debido a la columna de agua, se ha utilizado la densidad del agua de $1,00 e^{-5}$ $\mathrm{N} / \mathrm{mm}^{3}$. No se han considerado los traspasos de las chapas, pues, en estos casos, la espesura es mayor que la de sólo una chapa, y, por eso, la tensión es menor. Todos los resultados de tensión están en MPa $\left(\mathrm{N} / \mathrm{mm}^{2}\right)$ y el coeficiente de seguridad será determinado por la Teoría de Tensión Normal Máxima, también conocida como Teoría de Rankine, la misma utilizada por la norma ASME VIII división 1.

Las características del material Acero Inox Duplex son: Módulo de Elasticidad: 193.053 MPa; Coeficiente de Poisson: 0,28; Tensión de fluencia (Sy) = $515 \mathrm{MPa}$; Resistencia máxima a la tracción (Sut) = 710 MPa. El biorreactor ha sido dividido en anillos con anchura de las chapas en dimensiones comerciales de $1400 \mathrm{~mm}$. Las divisiones que aparecen en el biorreactor de la figura 2 representan los anillos en el primer análisis de tensiones.

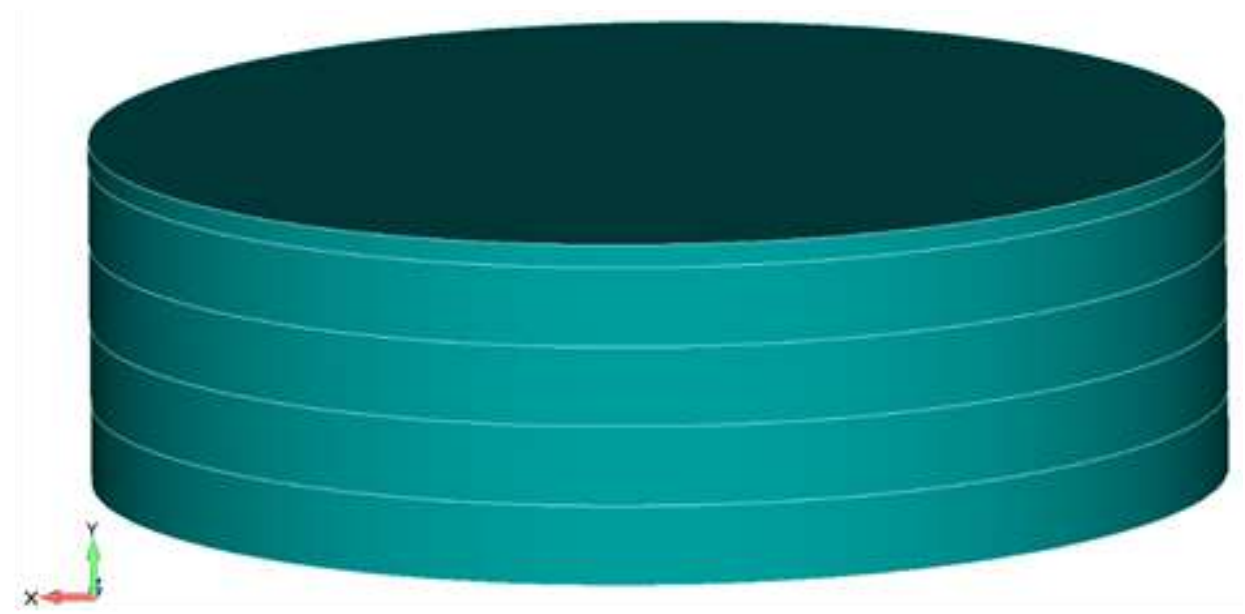

Fig. 2: Modelo de biorreactor en acero inox dúplex AISI 318. 
En la etapa de optimización dimensional, los cuatro primeros anillos tienen una espesura, las cuales serán las variables del proyecto. De esta forma, se tiene cuatro anillos que representan cuatro propiedades de espesura. El anillo superior de número 5 no entra en el proceso de optimización porque no se ha considerado la columna de líquido en el mismo. Para la fabricación del biorreactor dimensionado, el mismo se divide en anillos con anchuras comerciales de chapas de acero. Dichos anillos se unen por tornillos. Para optimizar los costos y conocer el comportamiento del tanque cuando está cargado, se hace necesaria su simulación con variación del espesor de las chapas.

La malla utilizada fue con elementos de cáscara isoparamétrica de 4 nudos, por lo tanto, de formulación linear. El primer análisis fue estático, y elementos con espesura de $8 \mathrm{~mm}$ como estimativa inicial, para verificar los niveles de tensión. Para malla de elementos finitos, se han utilizado 465.708 elementos y 465.133 nudos. El criterio para determinar el número de elementos ha sido el tamaño del elemento que será utilizado y, en este caso, los elementos de cáscara tienen la forma de cuadriláteros de cerca de $50 \mathrm{~mm}$ de cada lado. Con elementos tan pequeños, cuando son comparados a las dimensiones del tanque, se tiene una buena discretización de la geometría y los resultados del análisis de tensiones son más precisos.

\section{RESULTADOS Y DISCUSIÓN}

La primera simulación con chapas de $8 \mathrm{~mm}$ a lo largo del costado del biorreactor. La tensión en el biorreactor en su región crítica ha sido de $83,66 \mathrm{MPa}$ y, por lo tanto, con esta espesura de chapa, el coeficiente de seguridad $(n)$ sería de aproximadamente 6 veces. Como dicho coeficiente ha sido muy alto y por eso se puede optar por reducir la espesura de las chapas a la mitad, se hará un nuevo análisis, y los elementos tendrán espesura de 4,00 mm. Los resultados muestran que la tensión en el tanque es de cerca de 150,2 MPa lo que significa que, con esta espesura de chapa, el coeficiente de seguridad sería de 3,42. Ha admitido un valor de coeficiente de seguridad de 2,00 y el mayor valor de tensión admisible para el tanque debe ser de aproximadamente $250 \mathrm{MPa}$. Se ha utilizado un método de optimitización estructural y, con eso, los resultados de las espesuras de las chapas resultantes del proceso de optimización han sido: anillo 1: 4,00 mm; anillo 2: 2,00 mm; anillo 3: 1,33 mm; anillo 4: 1,33 mm. En la figura 3 se puede ver la simulación.

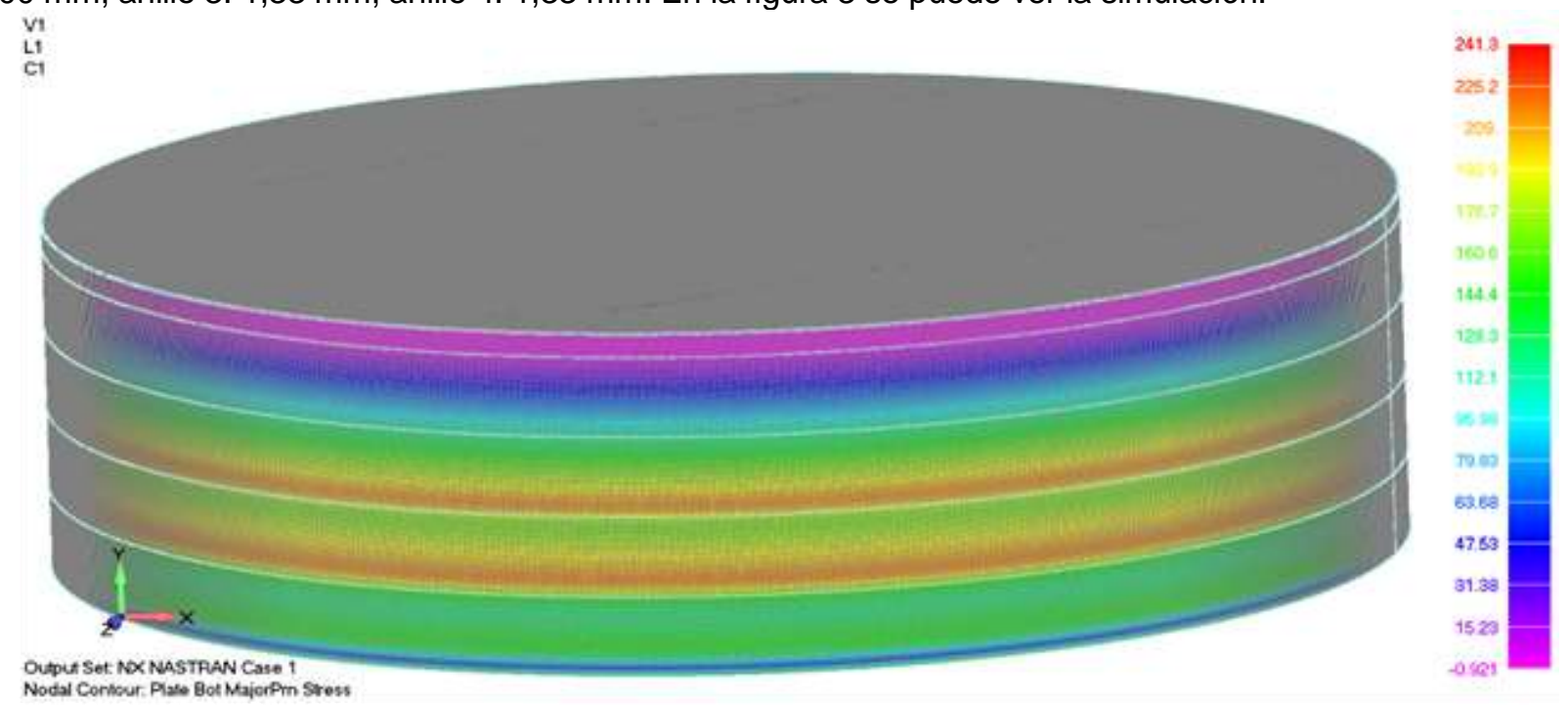

Fig. 3: Resultado de la simulación por elementos finitos. Utilización con chapas de 8mm de espesor (AISI 318).

Para optimización, se ha efectuado un análisis estático con la siguiente proposición de espesuras: anillo 1: 3,00 mm; anillo 2: 2,00 mm; anillo 3: 2,00 mm; anillo 4: 1,50 mm. Para tales espesuras se presenta el resultado del análisis de tensiones en la figura 4, en que se puede ver que las chapas que componen el primer anillo están más tensionadas en relación al resultado de optimización; pero, desde este resultado, la espesura está 1,00 mm menor y, con eso, se tendrá economia de material.

Cuantitativamente, se presenta en la figura 5, donde se verifica la tensión de 193, 29 MPa en un nudo de la malla, en el primer anillo del tanque, para la chapa de 3,00 mm de espesura. La mayor tensión ha sido encontrada en el segundo anillo y su valor de 236,1 MPa está dentro del valor de tensión admisible. Para la simulación de los demás aceros se tiene para A36: Módulo de Elasticidad: 199.948 MPa; Coeficiente de Poisson: 0,28; Tensión de Fluencia (Sy) = $250 \mathrm{MPa}$; Resistencia a la Máxima Tracción (Sut) = $350 \mathrm{MPa}$. Ya para el acero Inox 316 L: Módulo de Elasticidad: 193.053 MPa; Coeficiente de Poisson: 0,28; Tensión de Fluencia (Sy) = 240 MPa; Resistencia a la Máxima Tracción (Sut) = 320 MPa. 


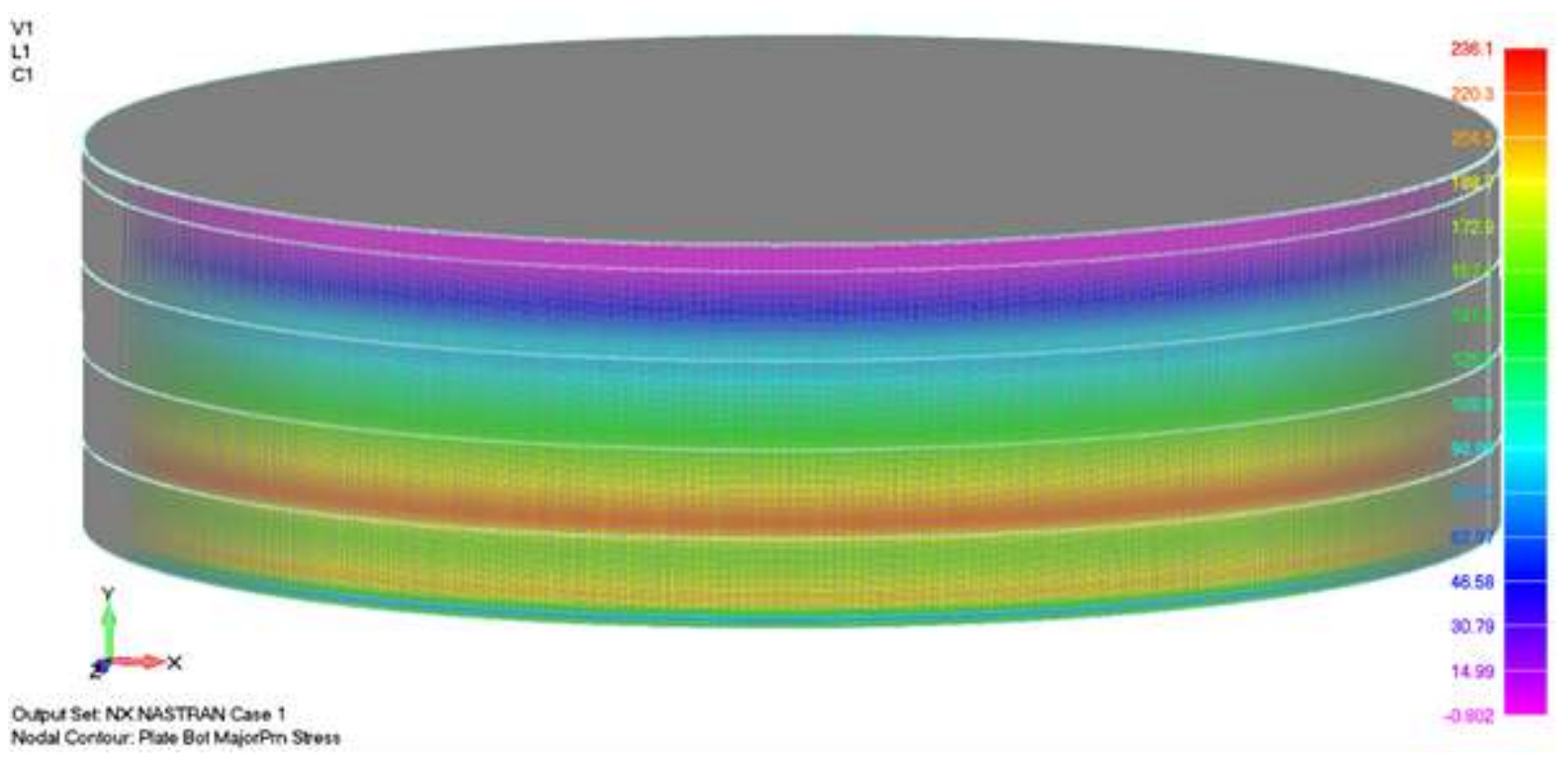

Fig. 4: Resultado simulación por elementos finitos. Utilización con chapas de espesor variado (AISI 318).

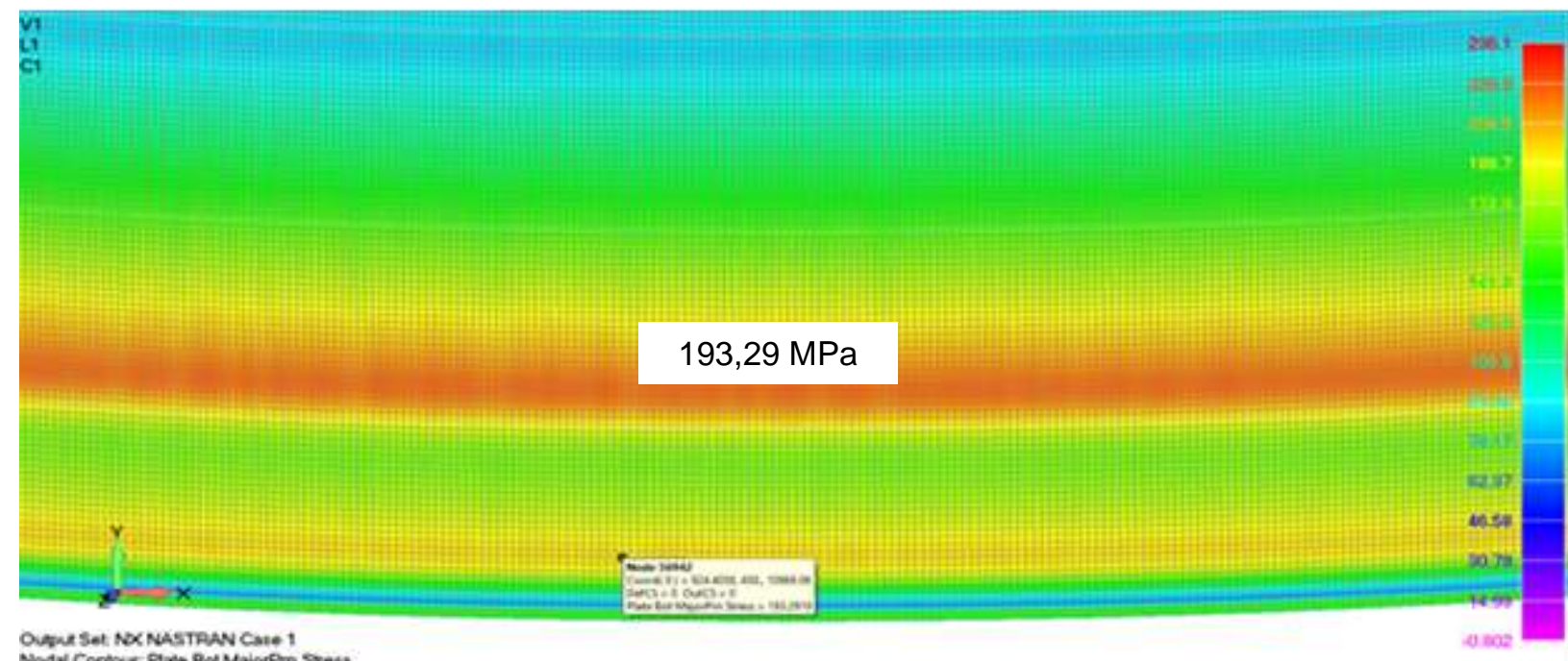

Fig. 5: Resultado simulación por elementos finitos. Región crítica para chapas de 3mm de espesor (AISI 318).

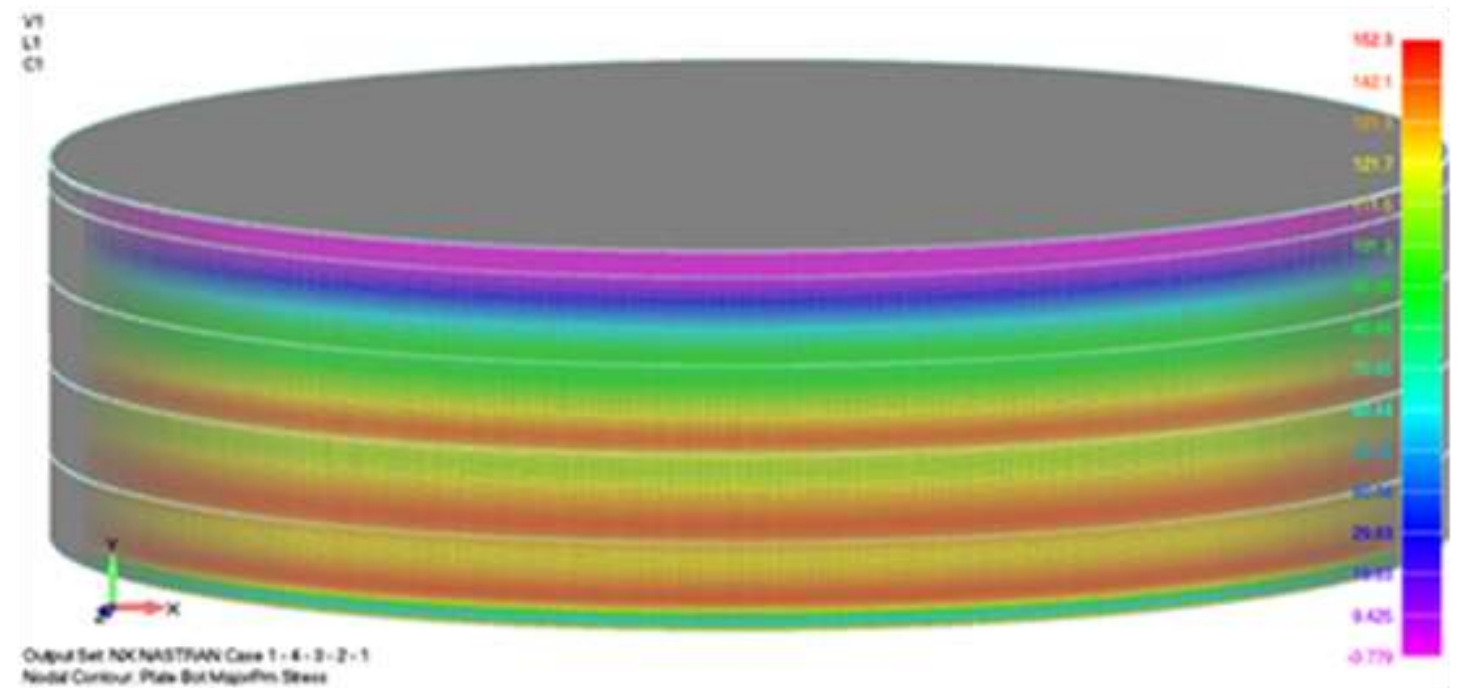

Fig. 6: Resultado simulación por elementos finitos. Utilización del acero ASTM A36 con 4mm de espesor. 
El análisis estático para todas las chapas con 4,00 mm de espesura ha aportado prácticamente los mismos valores de tensión ya esperados, porque el cálculo de tensión por elementos finitos utiliza el módulo de elasticidad y el coeficiente de Poison, y estos valores para el acero inox y aceros al carbón tienen diferencia inferior a un $1 \%$. Por lo tanto, los resultados de tensión podrían tener una variación inferior a un $1 \%$ si hubiesen sido hechos los análisis con cada uno de los materiales. Lo mismo se puede decir de los resultados de desplazamientos. Se puede ver la simulación en la figura 6.

Se puede optar por reducir la espesura de las chapas a la mitad, por eso se hará un nuevo análisis y los elementos tendrán espesura de 4,00 mm. Los resultados muestran que la tensión en el biorreactor es de cerca de 152,3 MPa y, por lo tanto, con esa espesura de chapa, el coeficiente de seguridad sería de cerca de 1,62. No se hace más necesario utilizar el método de optimización para determinar las espesuras de las chapas. Por eso, para el acero ASTM A36, ha sido propuesta una chapa de 3,00 mm al primer anillo, y una de espesura de 2,00 $\mathrm{mm}$ a los otros cuatro anillos. Puede verificarse que, para el acero duplex, se puede usar todas las chapas con 2,00 mm de espesura. Ya para el acero AISI $316 \mathrm{~L}$ y el ASTM A36 se debe usar los anillos con 4,00 mm de espesura, y para los demás, anillos con 2,00 mm de espesura. Los dos materiales tienen tensión de fluencia muy próxima, por eso es posible adoptar los valores: anillo 1: 4,00 mm; anillo 2: 3,00 mm; anillo 3: 2,00 mm; anillo 4: 1,50 mm.

\section{CONCLUSIONES}

A través de la simulación efectuada, puede verificarse la posibilidad de variación de las chapas en todo el biorreactor para los tres tipos de acero. Al comparar los tres tipos de acero, se ha observado mejor desempeño del acero AISI 318, al paso que, para la parte inferior, ha sido posible utilizar una espesura menor en el primer anillo, en comparación con otros materiales. Al utilizar este acero, se puede utilizar menos material, sin embargo es necesario verificar los costos relacionados. Los aceros AISI 316 L y ASTM A36 tuvieron un comportamiento semejante en la simulación. El acero AISI $316 \mathrm{~L}$ posee una mayor durabilidad, pero tiene un costo mayor que el ASTM A36.

\section{REFERENCIAS}

ASME - American Society of Mechanical Engineers. Boiler and Pressure Vessel Code, Section VIII, Division 1, New York, (2004)

Cervi, R. G., Esperancini, M. S. T., y Bueno, O. C. Viabilidad Económica de la Utilización de Biogás para la Conversión en Energía Eléctrica. Información Tecnológica, 22(4), 3-14. (2011)

Chablat, D; Bennis, F; Hoessler, B; Guilbert, M. Haptic devices and objects, robots and mannequin simulation in a CAD-CAM software: eM-Virtual Desktop. CoRR abs/1104.0834: Institut de Recherche en Communications et Cybernétique de Nantes - France (2011)

Coelho, M. A, A; Souza, J.; Schaeffer, L; Rossini, E. G. Study on the economic viability of hi-tech biogas plants. Revista Espacios. 35(3), 2-8 (2014)

Iglesias, L. y otros 5 autores, Biomethanization of municipal solid waste in a Pilot Plant. Water Research, 34(2), 447-454 (2000)

Guerra, R. L. Aplicación de la realidad virtual no inmersiva para Ingenieros Agrícolas. Universidad Agraria de La Habana. Revista Ciencias Técnicas Agropecuarias, 21(1), 68-72 (2012)

Morero, B. y Campanella, E. A Simulación del Proceso de Absorción Química con Soluciones de Aminas para la Purificación Biogás. Información Tecnológica, 24(1).25-32. (2013)

Oechsner, H., Lemmer, A. Was kann die Hydrolyse bei der Biogasvergärung leisten? pp. 37-46, VDIBerichte 2057, (2009)

Souza, J. Desenvolvimento de tecnologias para compressão de biogás. Dissertação de Mestrado. PPGE3M - Universidade Federal do Rio Grande do Sul - Porto Alegre - RS, Brasil, (2010)

Souza, J; Schaeffer, L. Sistema de compresión de biogas y biometano. Revista Información tecnológica.24(6), 3-8 (2013)

Souza, J; Schaeffer, L. Estudo para fabricação de cilindros especiais para biometano. Revista Liberato (Novo Hamburgo-RS), 11(15), 33-38 (2010)

Varnero, M. T., Carú, M., Galleguillos, K. y Achondo, P. Tecnologías disponibles para la Purificación de Biogás usado en la Generación Eléctrica. Información Tecnológica, .23(2) 31-40. (2012) 